\title{
Electron beams of cylindrically symmetric spin polarization
}

\author{
Yan Wang ${ }^{1}$ and Chun-Fang $\mathrm{Li}^{1,2 *}$ \\ ${ }^{1}$ Department of Physics, Shanghai University, \\ 99 Shangda Road, 200444 Shanghai, China and \\ ${ }^{2}$ State Key Laboratory of Transient Optics and Photonics, \\ Xi'an Institute of Optics and Precision Mechanics of CAS, 710119 Xi'an, China
}

(Dated: November 7, 2018)

\begin{abstract}
Cylindrically symmetric electron beams in spin polarization are reported for the first time. They are shown to be the eigen states of total angular momentum in the $z$ direction. But they are neither the eigen states of spin nor the eigen states of orbital angular momentum in that direction.
\end{abstract}

PACS numbers: 41.75.Fr, 41.90.+e

\footnotetext{
* Email address: cfli@shu.edu.cn
} 


\section{INTRODUCTION}

Both photon beams and electron beams can carry orbital angular momentum. In the case of photon beams, the orbital angular momentum is carried by the spiral wavefront 1]. Photon beams that carry orbital angular momentum have important applications [2] in micro-machines, atom manipulation, and quantum information. In the case of electron beams, the orbital angular momentum was also shown [3] to be carried by the spiral structure of the beam's wavefront when the spin polarization is uniformly distributed. Electron beams that carry orbital angular momentum were generated very recently in experiments [4, 5] by making use of techniques that are similar to those [6] used for generating photon beams of orbital angular momentum.

Apart from the orbital-angular-momentum photon beams which are approximately uniformly polarized [7], another kind of photon beams that are rotationally invariant in polarization, the so-called cylindrical-vector beams [8], were also predicted [9, 10] and experimentally generated [8, 11-13]. The cylindrical-vector photon beams have drawn much attention in diverse areas of application [14]. Now that the local state of quantum electron waves can be described in terms of the concept of spin polarization [15], an much more interesting question arises naturally as to whether there exist electron beams that are cylindrically symmetric in spin polarization. To the best of our knowledge, no theoretical investigations of such electron beams have been reported.

An electron beam of cylindrically symmetric spin polarization means that it can not be an eigen state of the spin operator in a particular direction, $\mathbf{n} \cdot \boldsymbol{\sigma}$, where $\boldsymbol{\sigma}$ is the vector of Pauli matrix and $\mathbf{n}$ is a fixed unit vector. Upon noticing that the cylindrical-vector photon beams are eigen states of the total angular momentum in the propagation direction [7, 16], denoted as the $z$ axis, our aim is therefore to look for free electron beams that are eigen states of total angular momentum in the $z$ direction, but not eigen states of $\sigma_{z}$. For this purpose, we will follow a method that is similar to the method [7, 16] for constructing the cylindrical-vector photon beams. Astonishingly, the results of our calculations reveal that so constructed free electron beams have spin skyrmion textures [17-19].

For simplicity only monochromatic beams are considered. The wave function in the position representation, $\Psi(\mathbf{x})$, is related to the wave function in the momentum representation, 
$\psi\left(k_{\rho}, \varphi\right)$, via

$$
\Psi(\mathbf{x})=\frac{1}{2 \pi} \int \psi\left(k_{\rho}, \varphi\right) e^{i \mathbf{k} \cdot \mathbf{x}} k_{\rho} d k_{\rho} d \varphi
$$

in circular cylindrical coordinates, where $\mathbf{k}=\mathbf{e}_{x} k_{\rho} \cos \varphi+\mathbf{e}_{y} k_{\rho} \sin \varphi+\mathbf{e}_{z} k_{z}$ and $k_{z}=\left(k^{2}-\right.$ $\left.k_{\rho}^{2}\right)^{1 / 2}$. In non-relativistic quantum frame, the Hamiltonian of free electrons is $H=\frac{\mathbf{p}^{2}}{2 \mu}$ when the spin is omitted. A complete set of mutually compatible observables in the monochromatic case can be chosen to be composed of $p_{z}$ and $L_{z}$, the $z$ components of the linear and orbital angular momenta, respectively. The corresponding complete orthonormal set of eigen functions in the momentum representation is given by

$$
\psi_{k_{z} m}\left(k_{\rho}, \varphi ; \kappa\right)=\frac{\delta\left(k_{\rho}-\kappa\right)}{i^{m} \sqrt{2 \pi \kappa}} e^{i m \varphi}, m=0, \pm 1, \pm 2 \ldots
$$

where $\kappa=\left(k^{2}-k_{z}^{2}\right)^{1 / 2}$. Substituting it into Eq. (11) yields the orthonormal eigen functions in the position representation,

$$
\Psi_{k_{z} m}(\mathbf{x} ; \kappa)=\sqrt{\frac{\kappa}{2 \pi}} J_{m}(\kappa r) e^{i m \phi} e^{i k_{z} z},
$$

where $J_{m}$ is the Bessel function of the first kind and $\mathbf{x}=\mathbf{e}_{x} r \cos \phi+\mathbf{e}_{y} r \sin \phi+\mathbf{e}_{z} z$. Obviously, the eigen values of $p_{z}$ are $k_{z}$ (we choose $\hbar=1$ ) and the eigen values of $L_{z}$ are $m$. They are the scalar non-diffractive beams [20] in the sense that the probability distribution does not change along with the propagation.

Equipped with the scalar basis (2), we proceed to construct spinor states that are cylindrically symmetric in spin polarization. In analogy with the case of photon beams, there exist two distinct configurations. The transverse component is either in radial direction or in azimuthal direction.

\section{TRANSVERSE COMPONENT IS IN RADIAL DIRECTION}

When the spin is taken into account, the free Hamiltonian is $H=\frac{(\boldsymbol{\sigma} \cdot \mathbf{p})^{2}}{2 \mu}$. We first introduce a unit-vector operator that is defined [7, 16] in terms of $\mathbf{p}$ and the unit vector $\mathbf{e}_{z}$ along the $z$ axis as $\mathbf{v}=\frac{\mathbf{p} \times \mathbf{e}_{z}}{\left|\mathbf{p} \times \mathbf{e}_{z}\right|}$. In the momentum representation, it shows up as $\mathbf{v}=-\mathbf{e}_{\varphi}$. Then we pay attention to operator $\boldsymbol{\sigma} \cdot \mathbf{v}$. Its normalized eigen spinors corresponding to eigen values $\sigma= \pm 1$ have the forms of

$$
\psi_{+1}=\frac{1}{\sqrt{2}}\left(\begin{array}{c}
1 \\
-i e^{i \varphi}
\end{array}\right), \psi_{-1}=\frac{1}{\sqrt{2}}\left(\begin{array}{c}
-i e^{-i \varphi} \\
1
\end{array}\right),
$$


respectively. It constitutes a complete set of mutually compatible observables together with $p_{z}$ and the total angular momentum $J_{z}=L_{z}+\frac{1}{2} \sigma_{z}$ in the $z$ direction. Their common normalized eigen functions in the momentum representation are proven to be

$$
\psi_{\sigma, k_{z} m}\left(k_{\rho}, \varphi ; \kappa\right)=\psi_{\sigma} \psi_{k_{z} m}\left(k_{\rho}, \varphi ; \kappa\right)
$$

The eigen value of $J_{z}$ is $j=m+\frac{\sigma}{2}$. Substituting Eq. (44) into Eq. (11) and replacing $m$ with $j-\frac{\sigma}{2}$, one obtains the corresponding normalized eigen functions in the position representation,

$$
\Psi_{\sigma, k_{z} j}(\mathbf{x} ; \kappa)=\sqrt{\frac{\kappa}{4 \pi}}\left(\begin{array}{c}
\sigma J_{j-\frac{1}{2}}(\kappa r) e^{i\left(j-\frac{1}{2}\right) \phi} \\
J_{j+\frac{1}{2}}(\kappa r) e^{i\left(j+\frac{1}{2}\right) \phi}
\end{array}\right) e^{i k_{z} z}
$$

Let us prove that the spin polarization in these states is indeed cylindrically symmetric. According to definition [15] $\mathrm{s}=\frac{\Psi^{\dagger} \sigma \Psi}{\rho}$, where $\rho=\Psi^{\dagger} \Psi$ is the probability density, the transverse and longitudinal components of the spin polarization are given by

$$
\begin{gathered}
\mathbf{s}_{\perp}=\frac{2 \sigma J_{j-\frac{1}{2}}(\kappa r) J_{j+\frac{1}{2}}(\kappa r)}{J_{j-\frac{1}{2}}^{2}(\kappa r)+J_{j+\frac{1}{2}}^{2}(\kappa r)} \mathbf{e}_{r}, \\
s_{z}=\frac{J_{j-\frac{1}{2}}^{2}(\kappa r)-J_{j+\frac{1}{2}}^{2}(\kappa r)}{J_{j-\frac{1}{2}}^{2}(\kappa r)+J_{j+\frac{1}{2}}^{2}(\kappa r)},
\end{gathered}
$$

respectively. They clearly show that (a) the transverse component is in radial direction and both the transverse and longitudinal components are rotationally invariant, indicating that the spin polarization is cylindrically symmetric; (b) both the transverse and longitudinal components do not change along with the propagation, so that the beam described by Eq. (5) is non-diffractive in spin-polarization distribution as well as in probability distribution.

Eq. (6a) means that the transverse component of spin polarization at $r=0$ vanishes. That is to say, the spin polarization at the center is always longitudinal. Based on the following asymptotic behavior of the Bessel function,

$$
J_{n}(x) \approx \frac{x^{n}}{2^{n} n !} \text { when } x \rightarrow 0 \text { for } n \geq 0,
$$

it is deduced from Eq. (하 $)$ that $\left.s_{z}\right|_{r=0}=1$ when $j \geq \frac{1}{2}$ and $\left.s_{z}\right|_{r=0}=-1$ when $j \leq-\frac{1}{2}$. In addition, when either equation $J_{j-\frac{1}{2}}(\kappa r)=0$ or equation $J_{j+\frac{1}{2}}(\kappa r)=0$ is satisfied, the spin polarization is also purely longitudinal. Furthermore, when equation $J_{j-\frac{1}{2}}^{2}(\kappa r)=J_{j+\frac{1}{2}}^{2}(\kappa r)$ holds, the spin polarization is purely transverse. Consequently, as $r$ increases continuously from the center, the spin polarization varies smoothly between transverse and longitudinal 
directions alternatively, starting from a purely longitudinal one. In Fig. 1 are schematically shown the spin-polarization distributions of non-diffractive beams of $j= \pm \frac{1}{2}$, where the value of $\kappa r$ is taken from 0 to 2.4048 , the first zero point of $J_{0}(\kappa r)$ at which the spin polarization is opposite to that at the center.
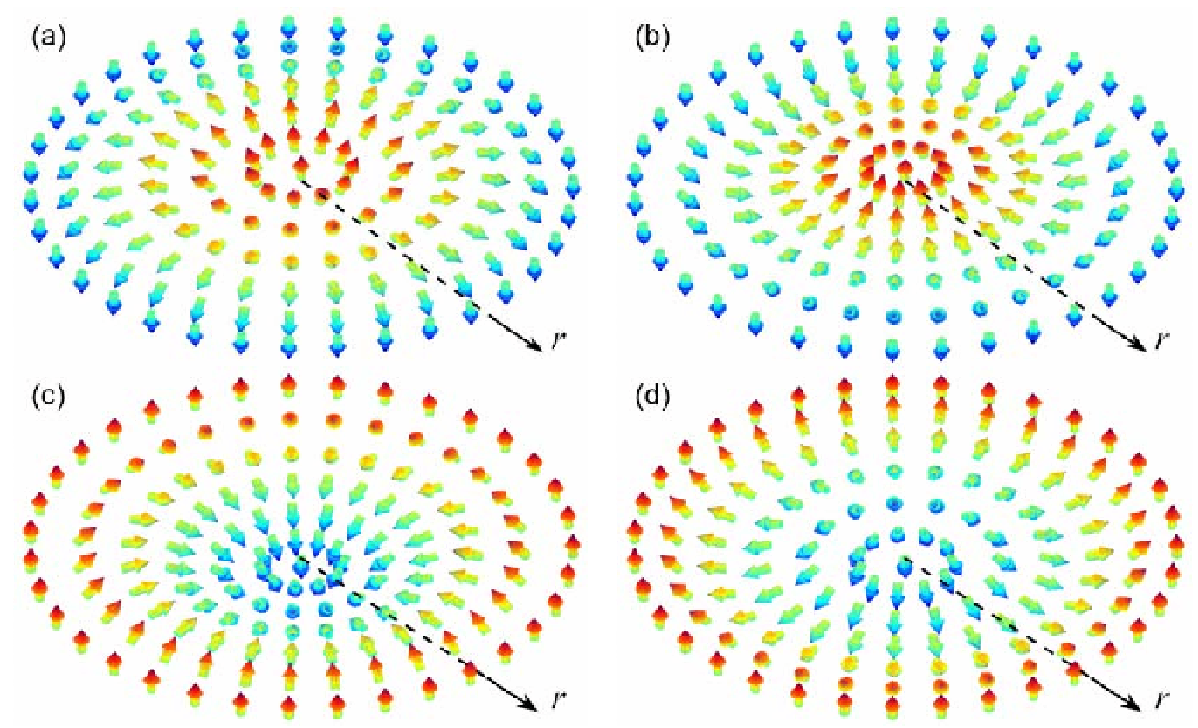

FIG. 1: (Color online) Schematic diagrams of the spin-polarization distributions for non-diffractive beams of (a) $j=\frac{1}{2}, \sigma=1$, (b) $j=\frac{1}{2}, \sigma=-1$, (c) $j=-\frac{1}{2}, \sigma=1$, and (d) $j=-\frac{1}{2}, \sigma=-1$.

Now we are ready to construct finite beams that are cylindrically symmetric in spin polarization. The eigen functions (4) or (5) constitute an orthonormal basis for monochromatic beams that have energy $\frac{k^{2}}{2 \mu}$. It is a direct calculation to show that the following wave functions in the momentum representation represent eigen states of $J_{z}$,

$$
\psi_{\sigma, m}\left(k_{\rho}, \varphi\right)=\int_{0}^{k} \sqrt{\kappa} f(\kappa) \psi_{\sigma, k_{z} m}\left(k_{\rho}, \varphi ; \kappa\right) d \kappa,
$$

which is $\frac{1}{i^{m} \sqrt{2 \pi}} f\left(k_{\rho}\right) \psi_{\sigma} e^{i m \varphi}$, where the scalar spectral function $f\left(k_{\rho}\right)$ is square integrable and satisfies the normalization condition $\int\left|f\left(k_{\rho}\right)\right|^{2} k_{\rho} d k_{\rho}=1$. As a superposition of eigen functions $\psi_{\sigma, k_{z} m}$ over the eigen value $k_{z}$, the wave function $\psi_{\sigma, m}$ is still an eigen state of operators $\boldsymbol{\sigma} \cdot \mathbf{v}$ and $J_{z}$. Substituting Eq. (7) into Eq. (11) and noticing the correspondence between Eqs. (4) and (5), one arrives at the corresponding wave functions in the position representation,

$$
\Psi_{\sigma, j}(\mathbf{x})=\sqrt{\frac{1}{4 \pi}}\left(\begin{array}{c}
\sigma F_{j-\frac{1}{2}}(r, z) e^{i\left(j-\frac{1}{2}\right) \phi} \\
F_{j+\frac{1}{2}}(r, z) e^{i\left(j+\frac{1}{2}\right) \phi}
\end{array}\right)
$$


where

$$
F_{n}(r, z)=\int_{0}^{k} f(\kappa) J_{n}(\kappa r) e^{i k_{z} z} \kappa d \kappa .
$$

Straightforward calculations give for the transverse and longitudinal components of spin polarization,

$$
\begin{aligned}
& \mathbf{s}_{\perp}=2 \sigma \frac{\operatorname{Re}\left(F_{j-\frac{1}{2}}^{*} F_{j+\frac{1}{2}}\right) \mathbf{e}_{r}+\operatorname{Im}\left(F_{j-\frac{1}{2}}^{*} F_{j+\frac{1}{2}}\right) \mathbf{e}_{\phi}}{\left|F_{j-\frac{1}{2}}(r, z)\right|^{2}+\left|F_{j+\frac{1}{2}}(r, z)\right|^{2}}, \\
& s_{z}=\frac{\left|F_{j-\frac{1}{2}}(r, z)\right|^{2}-\left|F_{j+\frac{1}{2}}(r, z)\right|^{2}}{\left|F_{j-\frac{1}{2}}(r, z)\right|^{2}+\left|F_{j+\frac{1}{2}}(r, z)\right|^{2}},
\end{aligned}
$$

respectively. Since function $F_{n}$ is axially symmetric, it is clearly seen from Eqs. (10) that the spin polarization is cylindrically symmetric. From Eqs. (9) and (10a) one may deduce that the spin polarization at $r=0$ has no transverse component. The same as before, whether it is in positive or negative $z$ direction depends on the sign of eigen value $j$. Furthermore, it can be seen from Eq. (9) that when the spectral function $f\left(k_{\rho}\right)$ is a real-valued function, $F_{n}$ is also real-valued at plane $z=0$, the plane of beam waist. In this case, the transverse component of spin polarization at plane $z=0$ is strictly in radial direction.

It is noted that the expectation of the spin in the state (8) vanishes,

$$
\int \Psi_{\sigma, j}^{\dagger}(\mathbf{x}) \boldsymbol{\sigma} \Psi_{\sigma, j}(\mathbf{x}) r d r d \phi=0
$$

This result can be understood as follows. Unit-vector operator $\mathbf{v}$ is an orbital vector operator in the sense [16] that it satisfies commutation relations $\left[L_{z}, v_{j}\right]=\sum_{k} i \epsilon_{z j k} v_{k}$, where $\epsilon_{i j k}$ is the Levi-Civitá pseudo tensor. So operator $\boldsymbol{\sigma} \cdot \mathbf{v}$ signifies a coupling between the spin and orbital angular momentum. Consequently, in its eigen state (86) there exists such a coupling which is introduced through the wave-vector dependence of the eigen spinors (3). As a result of this specific spin-orbit coupling $\boldsymbol{\sigma} \cdot \mathbf{v}$, we have the vanishing spin angular momentum.

As an example, we consider a paraxial beam which has a spectral function of Gaussian type, $f\left(k_{\rho}\right)=\sqrt{2} w_{0} \exp \left(-\frac{1}{2} w_{0}^{2} k_{\rho}^{2}\right)$, where $\frac{1}{k w_{0}}$ describes the divergence angle satisfying $k w_{0} \gg 1$. The integral formula

$$
\int_{0}^{\infty} e^{-a x^{2}} J_{\nu}(b x) x d x=\frac{\sqrt{\pi} b}{8 \sqrt{a^{3}}} e^{-\frac{b^{2}}{8 a}}\left\{I_{\frac{\nu-1}{2}}\left(\frac{b^{2}}{8 a}\right)-I_{\frac{\nu+1}{2}}\left(\frac{b^{2}}{8 a}\right)\right\}
$$

makes us to cope with the cases of $j \geq \frac{1}{2}$ and $j \leq-\frac{1}{2}$ separately, since it holds under the conditions [21] of $\operatorname{Re} \nu>-2$ as well as $\operatorname{Re} a>0$. Due to the relation $J_{-n}(x)=(-1)^{n} J_{n}(x)$, 
we will consider only the case of $j \geq \frac{1}{2}$ in the next of this section. Substituting $f\left(k_{\rho}\right)$ into Eq. (9), making use of paraxial approximation $k_{z} \approx k-\frac{\kappa^{2}}{2 k}$ in the factor $e^{i k_{z} z}$, and extending the upper integral limit to infinity, one finds

$$
F_{n}(r, z)=\frac{\sqrt{\pi} w_{0} r}{2 w^{3}} e^{-\frac{r^{2}}{4 w^{2}}}\left\{I_{\frac{n-1}{2}}\left(\frac{r^{2}}{4 w^{2}}\right)-I_{\frac{n+1}{2}}\left(\frac{r^{2}}{4 w^{2}}\right)\right\} e^{i k z},
$$

where $n=j \pm \frac{1}{2} \geq 0, w=w_{0}\left(1+i \frac{z}{z_{0}}\right)^{1 / 2}$, and $z_{0}=k w_{0}^{2}$. Since expression (11) contains factors of Gaussian and modified Bessel functions, the beams described by Eqs. (8) and (11) are referred to as modified-Bessel-Gaussian beams. In Fig. 2 are schematically shown the spin-polarization distributions at the plane of beam waist for (a) $j=\frac{1}{2}, \sigma=1$ and (b) $j=\frac{1}{2}, \sigma=-1$, where $\frac{r}{w_{0}}$ is taken from the center to 3.36 .
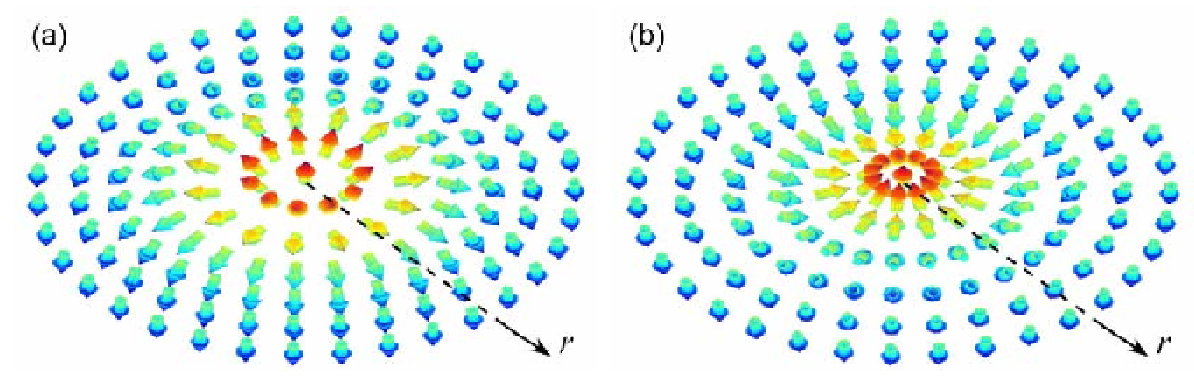

FIG. 2: (Color online) Schematic diagrams of the spin-polarization distributions for (a) $j=\frac{1}{2}$, $\sigma=1$ and (b) $j=\frac{1}{2}, \sigma=-1$.

An interesting phenomenon that can be seen from Fig. 2 as well as Eqs. (10) is that the distribution of spin polarization found here shows up as a skyrmion texture [17-19]. The spin polarization at a cross section of the beam is rotationally invariant about the center and varies smoothly along with the increase of the radius. Therefore the topological charge of such a skyrmion texture [22], $q=\frac{1}{2}\left(\left.s_{z}\right|_{r \rightarrow \infty}-\left.s_{z}\right|_{r=0}\right)$, is found from Eqs. (10b) and (11) to be

$$
q=-\frac{1}{2}\left(1+\frac{j}{j^{2}+1 / 4}\right)
$$

which does not change along with the beam propagation. It is dependent only on $j$, the quantum number of $J_{z}$. When $j=\frac{1}{2}$, we have $q=-1$ as is clearly shown in Fig. 2 , When $j \geq \frac{3}{2}$, the topological charge is a fractional number and approaches $-\frac{1}{2}$ in the limit $j \rightarrow+\infty$. 


\section{TRANSVERSE COMPONENT IS IN AZIMUTHAL DIRECTION}

Apart from the above mentioned beam the transverse spin polarization of which is in radial direction at the plane of beam waist, there exists another kind of beams the transverse spin polarization of which is in azimuthal direction at the same plane. For the sake of completeness, we briefly summarize the essential procedures of their construction and put forward the corresponding non-diffractive beams.

From unit-vector operator $\mathbf{v}$, we define another momentum-dependent unit-vector operator $\mathbf{u}=\mathbf{v} \times \frac{\mathbf{p}}{p}$. The eigen spinors of operator $\boldsymbol{\sigma} \cdot \mathbf{u}$ are as follows,

$$
\begin{aligned}
& \psi_{+1}^{u}=\frac{1}{\sqrt{2}}\left(\begin{array}{c}
\sqrt{1+w_{\rho}} \\
-e^{i \varphi} \sqrt{1-w_{\rho}}
\end{array}\right), \\
& \psi_{-1}^{u}=\frac{1}{\sqrt{2}}\left(\begin{array}{c}
e^{-i \varphi} \sqrt{1-w_{\rho}} \\
\sqrt{1+w_{\rho}}
\end{array}\right),
\end{aligned}
$$

which correspond to eigen values $\sigma= \pm 1$, respectively, where $w_{\rho}=\frac{k_{\rho}}{k}$. One may check that

$$
\psi_{\sigma, k_{z} m}^{u}\left(k_{\rho}, \varphi ; \kappa\right)=\psi_{\sigma}^{u} \psi_{k_{z} m}\left(k_{\rho}, \varphi ; \kappa\right)
$$

is the common eigen function of operators $\boldsymbol{\sigma} \cdot \mathbf{u}, p_{z}$, and $J_{z}$ in the momentum representation. The eigen value of $J_{z}$ is $j=m+\frac{\sigma}{2}$. Substituting Eq. (12) into Eq. (1) and replacing $m$ with $j-\frac{\sigma}{2}$, one obtains the corresponding eigen functions in the position representation,

$$
\begin{aligned}
& \Psi_{+1, k_{z} j}^{u}=\sqrt{\frac{\kappa}{4 \pi}}\left(\begin{array}{c}
\sqrt{1+w_{\kappa}} J_{j-\frac{1}{2}}(\kappa r) e^{i\left(j-\frac{1}{2}\right) \phi} \\
-i \sqrt{1-w_{\kappa}} J_{j+\frac{1}{2}}(\kappa r) e^{i\left(j+\frac{1}{2}\right) \phi}
\end{array}\right) e^{i k_{z} z}, \\
& \Psi_{-1, k_{z} j}^{u}=\sqrt{\frac{\kappa}{4 \pi}}\left(\begin{array}{c}
-i \sqrt{1-w_{\kappa}} J_{j-\frac{1}{2}}(\kappa r) e^{i\left(j-\frac{1}{2}\right) \phi} \\
\sqrt{1+w_{\kappa}} J_{j+\frac{1}{2}}(\kappa r) e^{i\left(j+\frac{1}{2}\right) \phi}
\end{array}\right) e^{i k_{z} z},
\end{aligned}
$$

for $\sigma= \pm 1$, respectively.

The transverse and longitudinal components of spin polarization in state $\Psi_{+1, k_{z} j}^{u}$ are given by

$$
\begin{aligned}
& \mathbf{s}_{\perp}^{u+}=-\frac{2 w_{z} J_{j-\frac{1}{2}}(\kappa r) J_{j+\frac{1}{2}}(\kappa r) \mathbf{e}_{\phi}}{\left(1+w_{\kappa}\right) J_{j-\frac{1}{2}}^{2}(\kappa r)+\left(1-w_{\kappa}\right) J_{j+\frac{1}{2}}^{2}(\kappa r)}, \\
& s_{z}^{u+}=\frac{\left(1+w_{\kappa}\right) J_{j-\frac{1}{2}}^{2}(\kappa r)-\left(1-w_{\kappa}\right) J_{j+\frac{1}{2}}^{2}(\kappa r)}{\left(1+w_{\kappa}\right) J_{j-\frac{1}{2}}^{2}(\kappa r)+\left(1-w_{\kappa}\right) J_{j+\frac{1}{2}}^{2}(\kappa r)},
\end{aligned}
$$


respectively, where $w_{z}=\frac{k_{z}}{k}$. And the transverse and longitudinal components of spin polarization in state $\Psi_{-1, k_{z} j}^{u}$ are given by

$$
\begin{aligned}
& \mathbf{s}_{\perp}^{u-}=\frac{2 w_{z} J_{j-\frac{1}{2}}(\kappa r) J_{j+\frac{1}{2}}(\kappa r) \mathbf{e}_{\phi}}{\left(1-w_{\kappa}\right) J_{j-\frac{1}{2}}^{2}(\kappa r)+\left(1+w_{\kappa}\right) J_{j+\frac{1}{2}}^{2}(\kappa r)} \\
& s_{z}^{u-}=\frac{\left(1-w_{\kappa}\right) J_{j-\frac{1}{2}}^{2}(\kappa r)-\left(1+w_{\kappa}\right) J_{j+\frac{1}{2}}^{2}(\kappa r)}{\left(1-w_{\kappa}\right) J_{j-\frac{1}{2}}^{2}(\kappa r)+\left(1+w_{\kappa}\right) J_{j+\frac{1}{2}}^{2}(\kappa r)}
\end{aligned}
$$

respectively. Obviously, the spin-polarization distributions in both situations are cylindrically symmetric. But now the transverse components are all in azimuthal direction. In much the same way as used before, one is easy to construct finite beams the transverse spin polarization of which is in azimuthal direction at the plane of beam waist.

\section{CONCLUSIONS AND DISCUSSIONS}

In a word, we have found solutions of quantum electron beams that bear close analogy to cylindrical-vector photon beams to show cylindrical symmetry in spin polarization. These beams might be generated in experiments that are designed specially in much the similar way to those [8, 11-13] that are designed to generate cylindrical-vector photon beams. For example, one might consider the transmission of uniformly polarized beams through a magnetic thin film that has a stable two-dimensional skyrmion texture in magnetization [19, 23, 24]. Another possibility might be the electron emission from a source that has a stable two-dimensional skyrmion texture in magnetization.

CFL is indebted to Ulrich K. Rößler for his helpful suggestions. This work was supported in part by the National Natural Science Foundation of China (60877055 and 60806041) and the Shanghai Leading Academic Discipline Project (S30105).

[1] L. Allen, M. W. Beijersbergen, R. J. C. Spreeuw, and J. P. Woerdman, Phys. Rev. A 45, 8185 (1992).

[2] S. Franke-Arnold, L. Allen, and M. Padgett, Laser Photon. Rev. 2, 299 (2008).

[3] K. Y. Bliokh, Y. P. Bliokh, S. Savel'ev, and F. Nori, Phys. Rev. Lett. 99, 190404 (2007).

[4] M. Uchida and A. Tonomura, Nature 464, 737 (2010). 
[5] J. Verbeeck, H. Tian, and P. Schattschneider, Nature 467, 301 (2010).

[6] M.W. Beijersbergen, R.P.C. Coerwinkel, M. Kristensen, and J.P. Woerdman, Opt. Commun. 112, 321 (1994).

[7] C.-F. Li, Phys. Rev. A 80, 063814 (2009).

[8] K. Youngworth and T. Brown, Opt. Express 7, 77 (2000).

[9] R. H. Jordan and D. G. Hall, Opt. Lett. 19, 427 (1994).

[10] D. G. Hall, Opt. Lett. 21, 9 (1996).

[11] T. V. Higgins, Laser Focus World 28, 18 (1992).

[12] M. Stalder and M. Schadt, Opt. Lett. 21, 1948 (1996).

[13] Y. Kozawa and S. Sato, Opt. Lett. 30, 3063 (2005).

[14] Q. Zhan, Adv. Opt. Photon. 1, 1 (2009).

[15] E. Merzbacher, Quantum Mechanics, 3rd Ed. (John Wiley \& Sons, New York, 1998).

[16] S. J. van Enk and G. Nienhuis, J. Mod. Opt. 41, 963 (1994).

[17] S.L. Sondhi, A. Karlhede, S.A. Kivelson, and E.H. Rezayi, Phys. Rev. B 47, 16419 (1993).

[18] A.N. Bogdanov and D.A. Yablonskii, Zh. Eksp. Teor. Fiz. 95, 178 (1989)[Sov. Phys. JETP 68, 101 (1989)]; A.N. Bogdanov and A. Hubert, J. Magn. Magn. Mater. 138, 255 (1994).

[19] X.Z. Yu et. al., Nature 465, 901 (2010).

[20] J.E. Durnin, J. Opt. Soc. Am. A 4, 651 (1987).

[21] I.S. Gradshteyn and I.M. Ryzhik, Table of integrals, series, and products, 7th Ed. (Elsevier, Amsterdam, 2007).

[22] K. Moon et. al., Phys. Rev. B 51, 5138 (1995).

[23] U.K. Rößler, A.N. Bogdanov, and C. Pfleiderer, Nature 442, 797 (2006).

[24] X.Z. Yu et. al., Nature Materials 10, 106 (2011). 